\title{
Defect formation and annealing behaviors of fluorine-implanted GaN layers revealed by positron annihilation spectroscopy
}

\author{
M. J. Wang, ${ }^{1}$ L. Yuan, ${ }^{1}$ C. C. Cheng, ${ }^{2}$ C. D. Beling, ${ }^{2}$ and K. J. Chen ${ }^{1, a)}$ \\ ${ }^{1}$ Department of Electronic and Computer Engineering, Hong Kong University of Science and Technology, \\ Clear Water Bay, Hong Kong \\ ${ }^{2}$ Department of Physics, The University of Hong Kong, Pok Fu Lam Road, Hong Kong
}

(Received 24 November 2008; accepted 22 January 2009; published online 11 February 2009)

\begin{abstract}
Defect formation and annealing behaviors of fluorine-implanted, unintentionally doped GaN layers were studied by positron annihilation spectroscopy (PAS). Single Ga vacancies $\left(V_{\mathrm{Ga}}\right)$ were identified as the main vacancy-type defects detected by PAS after fluorine implantation at $180 \mathrm{keV}$ with a dose of $1 \times 10^{15} \mathrm{~cm}^{-2}$. Implantation-induced $V_{\mathrm{Ga}}$ tend to aggregate and form vacancy clusters after postimplantation annealing in $\mathrm{N}_{2}$ ambient at $600{ }^{\circ} \mathrm{C}$. Fluorine ions tend to form F-vacancy complexes quickly after thermal annealing, which is consistent with the proposed diffusion model that predicts the behaviors of fluorine in GaN. (C) 2009 American Institute of Physics.
\end{abstract}

[DOI: 10.1063/1.3081019]

The incorporation of fluorine $(\mathrm{F})$ ions by low-energy plasma ion implantation into $\mathrm{AlGaN} / \mathrm{GaN}$ heterostructures is an effective low-cost approach to achieving postepitaxy threshold voltage modulation in AlGaN/GaN high-electron mobility transistors (HEMTs). ${ }^{1}$ The F ions effectively deplete the high density two-dimensional electron gases in the transistor channels and shift the threshold voltage by as much as $5 \mathrm{~V}$ to positive values to enable the realization of self-aligned enhancement-mode devices that are preferred in many applications. ${ }^{2,3}$ Promising reliability results have been reported, suggesting good stability of the F ions in III-nitride materials. ${ }^{4}$ Although the behaviors of $\mathrm{F}$ ions in Si have been investigated extensively, ${ }^{5}$ the underlying physical mechanisms that govern the incorporation, diffusion, and stabilization of $\mathrm{F}$ ions in group-III nitride semiconductors remain mostly undescribed. For example, there is a lack of experimental evidence that reveals how implantation-induced defects are generated and recovered, how the dynamic F diffusion process works, and what interactions of $\mathrm{F}$ with the defects are. Understanding these mechanisms would likely provide helpful information on the optimization of the process parameters such as the plasma power, annealing temperature, etc. ${ }^{1}$

In this letter, we report on our investigations of defect formation and annealing behaviors of F-implanted GaN layers using positron annihilation spectroscopy (PAS) and secondary ion mass spectroscopy (SIMS). We found that implantation-induced single $\mathrm{Ga}$ vacancies $\left(V_{\mathrm{Ga}}\right)$ tend to accumulate and form vacancy clusters after postimplantation annealing in $\mathrm{N}_{2}$. We also found that $\mathrm{F}$ ions tend to occupy $\mathrm{Ga}$ vacancies, which supports the diffusion mechanism that has been modeled based on molecular dynamic (MD) simulations.

The unintentionally doped GaN sample used in the present experiment was grown on (0001) sapphire substrates by metal-organic chemical vapor deposition (MOCVD) at $1070{ }^{\circ} \mathrm{C}$. The background electron density and mobility were measured to be $2 \times 10^{16} \mathrm{~cm}^{-3}$ and $400 \mathrm{~cm}^{2} / \mathrm{V} \mathrm{s}$, respectively, in room-temperature Hall effect measurements.

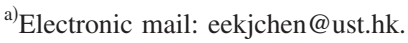

${ }^{19} \mathrm{~F}^{+}$ions were implanted into the GaN layers at $180 \mathrm{keV}$ with a dose of $1 \times 10^{15} \mathrm{~cm}^{-2}$ using a Varian CF3000 ion implanter. A separate experiment was performed by implanting low-energy $(10 \mathrm{keV}){ }^{19} \mathrm{~F}^{+}$ions at a dose of 1.5 $\times 10^{13} \mathrm{~cm}^{-2}$ into the channel region of an $\mathrm{AlGaN} / \mathrm{GaN}$ heterostructure to evaluate the charge state of the $\mathrm{F}$ ions after implantation. A shift of about $+3 \mathrm{~V}$ in the threshold voltage was observed, suggesting that the charge state of the F ions changed to negative after they were implanted into $\mathrm{GaN}$ because of the strong electronegativity of F. After implantation, the sample was cut into small pieces and furnace annealed in $\mathrm{N}_{2}$ ambient at $600{ }^{\circ} \mathrm{C}$ for times ranging from $30 \mathrm{~s}$ to $72 \mathrm{~h}$. Time-of-flight secondary-ion mass spectrometry (TOFSIMS) was performed to determine the concentration-depth profile of the $\mathrm{F}$ ions in the as-implanted and annealed samples. A piece of the as-grown sample was used as the reference. A slow positron beam with energy ranging from 0.35 to $18 \mathrm{keV}$ was used for the PAS study. The broadening Doppler spectra of the annihilation radiation were measured with a Ge detector as a function of the incident positron energy $E$. The $S$ parameter was defined as the central fraction of the annihilation lines over the energy range of $511 \pm 1.62 \mathrm{keV}$. The high-momentum part was characterized by the $W$ parameter, which was determined from the tail of the peak in the range of $2.55 \mathrm{keV} \leq\left|\Delta E_{\gamma}\right| \leq 5.11 \mathrm{keV}$, where $\Delta E_{\gamma}$ represents the energy offset from the peak (511 keV).

The measured $S$ - $E$ curves of the as-grown and asimplanted samples are plotted in Fig. 1(a), together with the fluorine distribution profile measured by TOF-SIMS. The mean implantation depth of the positrons in $\mathrm{GaN}$ is about $790 \mathrm{~nm}$ at $20 \mathrm{keV}$. The F implantation results in larger $S$, indicating the generation of a large number of vacancies, consistent with the MD simulation results. ${ }^{6}$ A nitrogen vacancy is much smaller than a Gacancy and nitrogen vacancy is supposed to be positively charged in $n$-type GaN. ${ }^{7}$ Thus, the increased $S$ parameter is the result of the negatively charged $\mathrm{Ga}$ vacancies induced by ion implantation.

Figure 1(b) plots the $W-S$ relations in the as-grown and as-implanted samples. The characteristic points of the surface and bulk follow the same straight line in the as-grown 


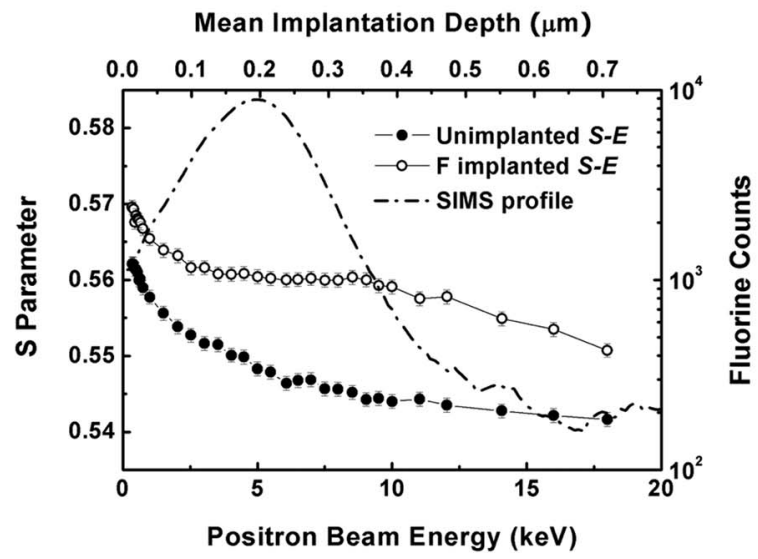

(a)

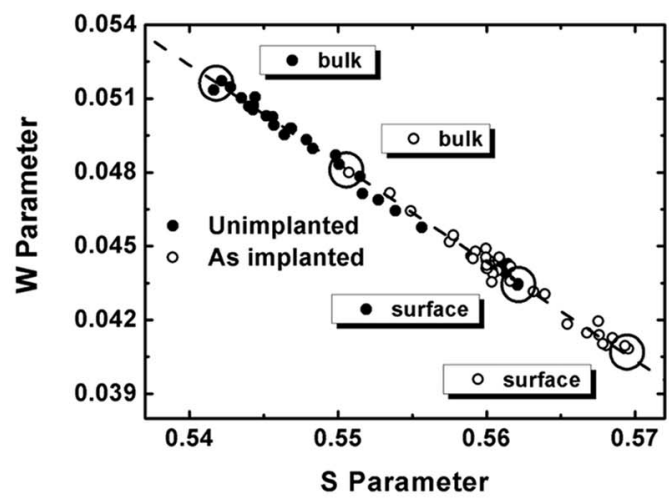

(b)

FIG. 1. (a) $S$-E curves of the GaN layer before and after F ion implantation. The depth profile of fluorine after implantation is also shown for comparison. (b) $W-S$ plots for as-grown and as-implanted samples.

and as-implanted samples. Some earlier studies provided evidence of positrons being trapped in the $\mathrm{Ga}$ vacancies in MOCVD-grown GaN. ${ }^{8}$ The straight line of the $W-S$ plot indicates that the surface states and Ga vacancies (mainly single vacancies) are the two dominant annihilation sites in the as-grown sample. ${ }^{9}$ The $(W, S)$ relation observed in the as-implanted sample follows the same line, suggesting that the same type of Ga vacancy is created during F implantation as detected by positron annihilation.

Figure 2(a) shows the $S-E$ curve of the F-implanted samples annealed in $\mathrm{N}_{2}$ ambient at $600{ }^{\circ} \mathrm{C}$ for times ranging from $30 \mathrm{~s}$ to $72 \mathrm{~h}$. The $\mathrm{F}$ distribution profiles of these samples are shown in Fig. 2(b). The $S$ parameter increases with longer annealing time, especially in the region around the projected range of the $\mathrm{F}$ ions, while it maintains relatively small values in the tail region. Previous reports suggest that it is difficult to remove defects induced by ion implantation fully in GaN because of the high critical annealing temperature. ${ }^{10}$ The gradually increasing $S$ parameter indicates that Ga divacancies, trivacancies, and vacancy clusters may be generated as a result of the aggregation of single vacancies during annealing. ${ }^{11,12}$ The aggregation of the single vacancies is a slow process, as evidenced by the continuous increase in the $S$ parameter value with the increasing annealing time.

The $W$-S characteristics measured in as-grown, asimplanted, and annealed samples are plotted in Fig. 3. The $(W, S)$ values of the annealed samples deviate from the
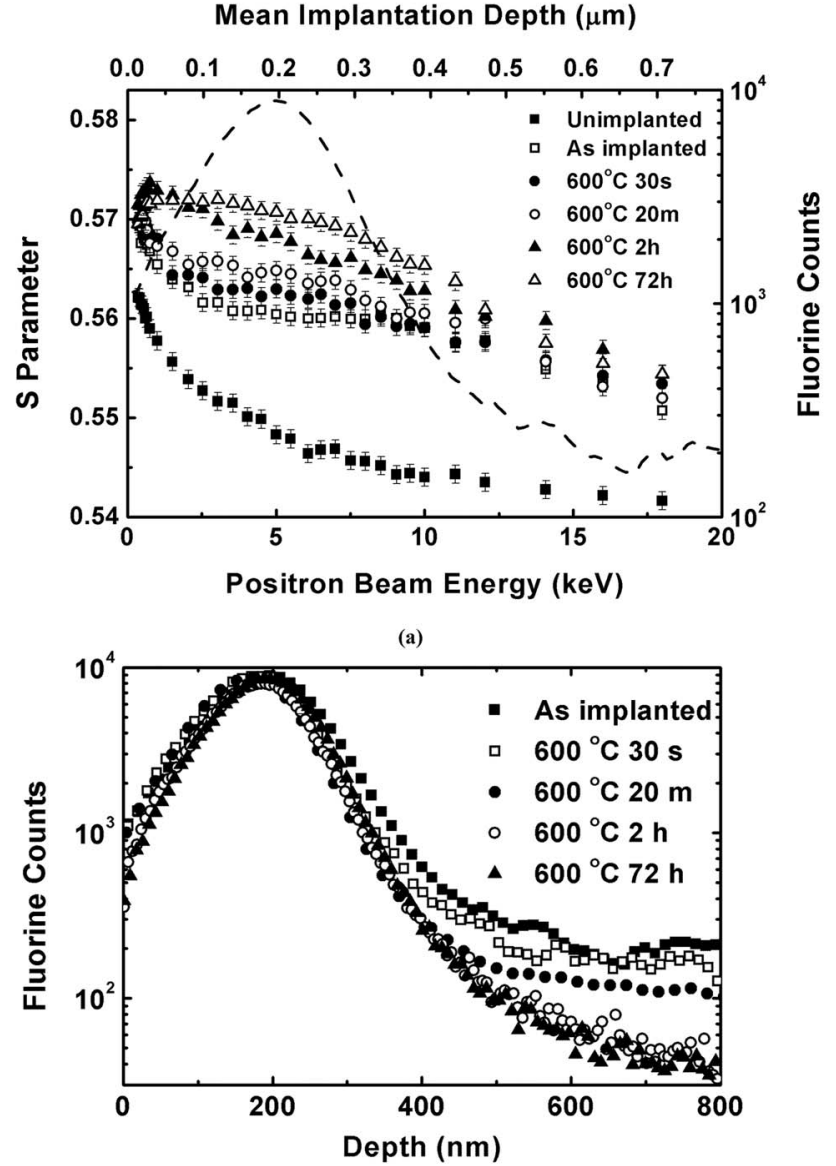

(b)

FIG. 2. (a) $S-E$ curves of the fluorine implanted samples after annealing in $\mathrm{N}_{2}$ atmosphere at $600{ }^{\circ} \mathrm{C}$ from $30 \mathrm{~s}$ to $72 \mathrm{~h}$. (b) SIMS profiles of fluorine implanted into GaN layer before and after annealing.

straight line obtained in the as-grown and as-implanted samples. This deviation increases with increasing annealing time. In the samples with long annealing times ( 2 and $72 \mathrm{~h}$ ), starting from the surface, the $W-S$ characteristics are significantly different from the straight line that characterizes the behavior of the as-implanted sample, providing evidence of the formation of new types of vacancies. In the region deep inside the bulk, the $W-S$ relation shifts back to the bulk state

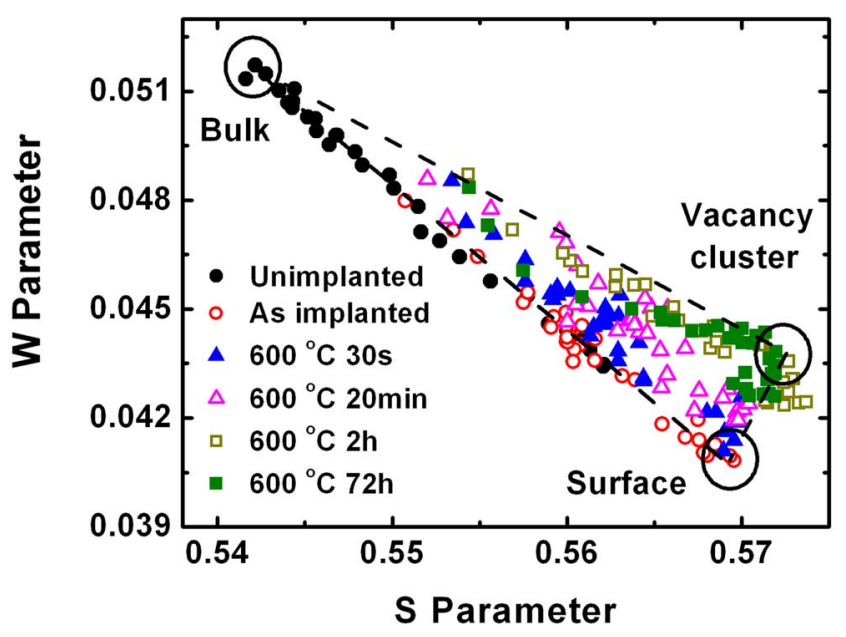

FIG. 3. (Color online) $W-S$ plots of the samples before and after annealing. 


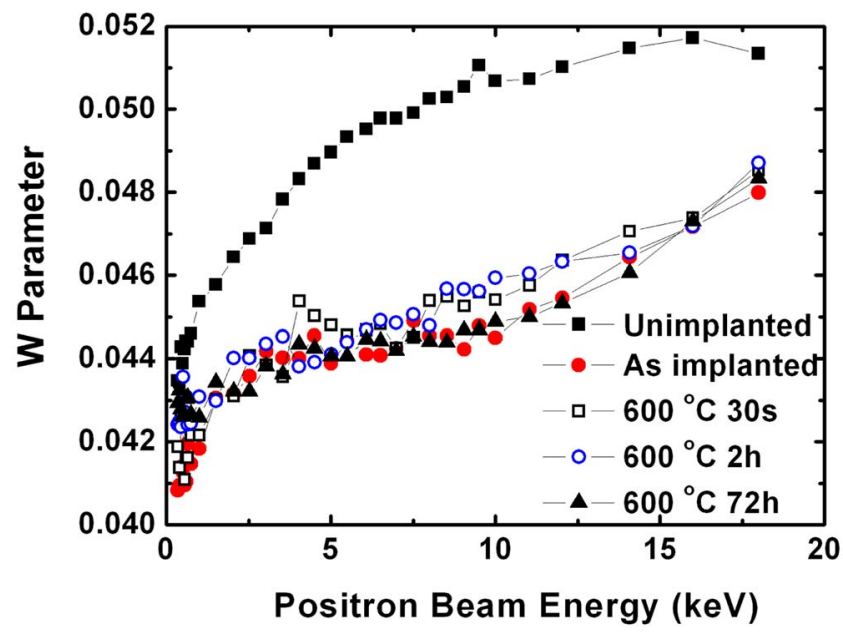

FIG. 4. (Color online) $W$-E curves of the as-grown, as-implanted, and thermal annealed samples. The curve of the sample annealed for $20 \mathrm{~min}$ is not shown for clarity. It should be located between the samples annealed for $30 \mathrm{~s}$ and $2 \mathrm{~h}$.

of the as-implanted samples, suggesting that no new vacancy is formed. In fact, the $W-S$ relations of the samples with long annealing times fall along two straight lines: the first linking the characteristic point of the surface and the vacancy cluster and the second linking the vacancy cluster and the bulk state. We conclude that three distinctive annihilation processes exist in the surface, vacancy clusters, and the bulk (single vacancy). The $W-S$ relations in the samples with short to medium annealing times lie in the triangle formed by the three straight lines, suggesting that other defects such as $\mathrm{Ga}$ divacancies or trivacancies may be generated during the annealing process.

Figure 4 shows the $W-E$ curves of the as-grown, asimplanted, and annealed samples. A decrease in the $W$ parameter after implantation corresponds to an increase in the $S$ parameter caused by the large number of $\mathrm{Ga}$ vacancies induced during implantation. Figure 2(a) shows that the $S$ parameter increases after annealing due to the generation of new types of vacancies with large open volumes. Thus, the $W$ parameter would be expected to decrease after annealing if these vacancy clusters do not incorporate any other atoms. However, a slight increase in the $W$ parameter is observed in the samples annealed for $30 \mathrm{~s}$ and $2 \mathrm{~h}$. The simultaneous increases in $W$ and $S$ in the annealed samples suggest that vacancy agglomeration is coupled with the formation of vacancy-F complexes. The $\mathrm{F}$ ions incorporated in the vacancy clusters present positrons with high-momentum electrons in the F atom's outermost shell. ${ }^{5,13}$ Thus, we conclude that $\mathrm{F}$ ions tend to diffuse and form F-vacancy complexes after annealing.

According to recent MD calculations, ${ }^{14}$ the diffusion potential energy barrier of fluorine in $\mathrm{GaN}$ between an interstitial site $(I)$ and a $\mathrm{Ga}$ vacancy $\left(V_{\mathrm{Ga}}\right)$ site is the lowest at $1.4 \mathrm{eV}$, while that between the $I$ site and a nitrogen vacancy $\left(V_{\mathrm{N}}\right)$ is $2.1 \mathrm{eV}$. The potential energy barrier between two adjacent $I$ sites is the highest at $5 \mathrm{eV}$. Thus, a Ga-vacancy assisted diffusion model, rather than a N-vacancy assisted diffusion or an interstitial-interstitial diffusion model, is proposed to describe the surface-oriented outdiffusion of fluorine in GaN after implantation, as shown in Fig. 2(b), which is consistent with the F-vacancy complex observations by PAS. The increase in the $W$ parameter in samples annealed for $30 \mathrm{~s}$ indicates that the process should be quick, which is consistent with the quick outdiffusion of the $\mathrm{F}$ ions after short annealing, as shown in Fig. 2(b). The $W$ parameter decreases after $72 \mathrm{~h}$ thermal annealing. This is because the vacancy clusters continue to expand while the number of fluorine ions accommodated by the vacancies is saturated. Thus, the fraction of annihilation with low-momentum electrons goes up and the fraction of annihilation with highmomentum electrons goes down. However, the value of the $W$ parameter around the projected range of $\mathrm{F}$ ions is close to that in the as-implanted sample, which should be regarded as the characteristic of $\mathrm{F}$ ions.

In summary, we have studied the vacancy formation and evolution in F-implanted GaN by PAS and TOF-SIMS experiments. Single Ga vacancies are induced by F ion implantation and vacancy clusters are formed by long-term thermal annealing at $600{ }^{\circ} \mathrm{C}$. Evidence suggests the formation of F-vacancy complexes in $\mathrm{GaN}$ following $\mathrm{F}$ implantation and annealing. We suggest that $\mathrm{F}$ ions tend to form F-vacancy complexes quickly after thermal annealing. These results provide a framework in which to understand the behaviors of $\mathrm{F}$ in GaN-based electronic devices such as the AlGaN/GaN HEMT.

The authors are grateful to Dr. F. J. Xu and Professor B. Shen of Peking University for providing the GaN wafer used in this work. This work was supported by the Hong Kong Research Grant Council under the competitive earmarked research Grant No. 611706.

${ }^{1}$ Y. Cai, Y. G. Zhou, K. M. Lau, and K. J. Chen, IEEE Trans. Electron Devices 53, 2207 (2006)

${ }^{2}$ Y. Cai, Z. Q. Cheng, C. W. Tang, K. M. Lau, and K. J. Chen, IEEE Trans. Electron Devices 53, 2223 (2006).

${ }^{3}$ Y. Cai, Z. Q. Cheng, Z. C. Yang, C. W. Tang, K. M. Lau, and K. J. Chen, IEEE Electron Device Lett. 28, 328 (2007).

${ }^{4}$ C. W. Yi, R. N. Wang, W. Huang, C. W. Tang, K. M. Lau, and K. J. Chen, Technical Digest of International Electron Device Meeting, 2007, p. 389 (unpublished).

${ }^{5}$ X. D. Pi, C. P. Burrows, and P. G. Coleman, Phys. Rev. Lett. 90, 155901 (2003).

${ }^{6}$ L. Yuan, M. J. Wang, and K. J. Chen, Appl. Phys. Lett. 92, 102109 (2008).

${ }^{7}$ J. Neugebauer and C. G. Van de Walle, Appl. Phys. Lett. 69, 503 (1996).

${ }^{8}$ K. Saarinen, T. Laine, S. Kuisma, J. Nissilä, P. Hautojärvi, L. Dobrzynski,

J. M. Baranowski, K. Pakula, R. Stepniewski, M. Wojdak, A. Wysmolek,

T. Suski, M. Leszczynski, I. Grzegory, and S. Porowski, Phys. Rev. Lett. 79, 3030 (1997).

${ }^{9}$ R. Krause-Rehberg and H. S. Leipner, Positron Annihilation in Semiconductors: Defect Studies (Springer, Berlin, 1999).

${ }^{10}$ S. O. Kucheyev, J. S. Williams, and S. J. Pearton, Mater. Sci. Eng. R. 33, 51 (2001).

${ }^{11}$ K. Saarinen, P. Hautojärvi, and C. Corbel, in Identification of Defects in Semiconductors, edited by M. Stavola (Academic, New York, 1998), p. 234.

${ }^{12}$ A. Uedono, K. Ito, H. Nakamori, K. Mori, Y. Nakano, T. Kachi, S. Ishibashi, T. Ohdaira, and R. Suzuki, J. Appl. Phys. 102, 084505 (2007).

${ }^{13}$ A. Uedono, T. Kitano, K. Hamada, T. Moriya, T. Kawano, S. Tanigawa, R. Suzuki, T. Ohdaira, and T. Mikado, Jpn. J. Appl. Phys., Part 1 36, 2571 (1997).

${ }^{14}$ L. Yuan, M. J. Wang, and K. J. Chen, J. Appl. Phys. 104, 116106 (2008). 